\title{
GC-MS-based metabolomic analysis of human papillary thyroid carcinoma tissue
}

\author{
MINJIAN CHEN ${ }^{1-3^{*}}$, MEIPING SHEN $^{4 *}$, YANYUN LI $^{1}$, CUIPING LIU $^{4}$, KUN ZHOU $^{2,3}$, \\ WEIYUE $\mathrm{HU}^{2,3}$, BO XU ${ }^{1-3}$, YANKAI XIA ${ }^{2,3}$ and WEI TANG ${ }^{1,5}$ \\ ${ }^{1}$ Department of Endocrinology, Jiangyin People's Hospital, School of Medicine, Southeast University, \\ Jiangyin, Jiangsu 214400; ${ }^{2}$ State Key Laboratory of Reproductive Medicine, Institute of Toxicology; \\ ${ }^{3}$ Key Laboratory of Modern Toxicology of Ministry of Education, School of Public Health, Nanjing Medical University, \\ Nanjing, Jiangsu 211166; ${ }^{4}$ Department of General Surgery, The First Affiliated Hospital of Nanjing Medical University, \\ Nanjing, Jiangsu 210036; ${ }^{5}$ Department of Endocrinology, Jiangsu Province Official Hospital, \\ Nanjing, Jiangsu 210024, P.R. China
}

Received April 1, 2015; Accepted October 7, 2015

DOI: $10.3892 / \mathrm{ijmm} .2015 .2368$

\begin{abstract}
Papillary thyroid carcinoma (PTC) is the most common type of thyroid cancer. Elucidating the molecular network that is altered in PTC may lead to the identification of the critical insight into the pathogenesis of PTC. Thus far, little is known regarding the global metabolomic alterations of PTC. Gas chromatography coupled with mass spectrometry-based metabolomics was used to analyze metabolomic alterations in matched PTC and normal thyroid tissues obtained from the patients. Multivariate statistical analyses were employed to determine the significant metabolomic differences. The mRNA levels of the associated metabolic enzyme genes were further assayed with reverse transcription-quantitative polymerase chain reaction analysis. Principal component analysis, partial
\end{abstract}

Correspondence to: Professor Wei Tang, Department of Endocrinology, Jiangyin People's Hospital, School of Medicine, Southeast University, 163 Shoushan Road, Jiangyin, Jiangsu 214400, P.R. China

E-mail: drtangwei@aliyun.com

${ }^{*}$ Contributed equally

Abbreviations: PTC, papillary thyroid carcinoma; NMR, nuclear magnetic resonance; GC, gas chromatography; MS, mass spectrometry; BSTFA, Bis-(trimethylsilyl)trifluoroacetamide; TMCS, trimethylchlorosilane; OPLS-DA, orthogonal partial leastsquares discriminate analysis; VIP, variable influence on projection; PCA, principal component analysis; PLS-DA, partial least-squares discriminant analysis; PHGDH, phoshphoglycerate dehydrogenase; G6PD, glucose-6-phosphate dehydrogenase; PGK1, phosphoglycerate kinase 1; LDHA, lactate dehydrogenase A; PTGS2, prostaglandinendoperoxide synthase 2; NAPDH, nicotinamide adenine dinucleotide phosphate; RNR, ribonucleotide reductase

Key words: metabolomics, papillary thyroid carcinoma, gas chromatography coupled with mass spectrometry least-squares discriminant analysis (PLS-DA) and orthogonal PLS-DA models were established, which could clearly separate human normal thyroid and PTC samples, and identified that metabolites in carbohydrate metabolism, including glucose, fructose, galactose, mannose, 2-keto-D-gluconic acid and rhamnose, consistently decreased, while metabolites in nucleotide metabolism, including malonic acid and inosine, and lipid metabolism, including cholesterol and arachidonic acid, significantly altered in PTC. Furthermore, the mRNA levels of metabolic enzyme genes, including glucose-6-phosphate dehydrogenase, phosphoglycerate kinase 1 , lactate dehydrogenase A, phosphoglycerate dehydrogenase and prostaglandin-endoperoxide synthase 2, significantly increased in PTC. Based on the metabolomic and mRNA data, various metabolites may be used for increased synthesis of nucleotides and oncogenic lipids in PTC, which may contribute to the pathogenesis of PTC. The present study provides a new understanding of the dysregulated metabolism in PTC and identifies potential avenues for the therapeutic intervention for this disease.

\section{Introduction}

Papillary thyroid carcinoma (PTC) is the most common type of thyroid cancer, which represents $75-85 \%$ of all thyroid cancer cases. Metabolomics is a newly emerging technology that holds promise for the diagnosis of disease and discovery of mechanisms linked to disease processes, particularly in cancers (1-4). Cancer cells have fundamentally altered cellular metabolism, which directly contributes to tumorigenicity and malignancy. Deciphering the molecular networks that are altered in PTC may lead to the identification of the critical insight into the pathogenesis of PTC. Although PTC is an important cancer with regard to research, little is known regarding the global metabolomic alterations of PTC. Currently, the nuclear magnetic resonance spectroscopy (NMR)-based metabolomic technique has been widely used in studies regarding thyroid carcinomas particularly in the diagnosis (5-7); however, the metabolic pathways that drive tumorigenesis in PTC remain to be elucidated. 
NMR is non-destructive, highly selective and useful in metabolite structural identification; however, it is limited by relatively lower sensitivities. By contrast, gas chromatography (GC) coupled with mass spectrometry (MS) offers a good combination of sensitivity and selectivity, which makes GC-MS an important tool in metabolomics. However, to the best of our knowledge, there are no published GC-MS based metabolomic studies on PTC.

Currently, integration of multiple layers of information is promising for acquiring a precise understanding of disease. The combination of metabolomic results and metabolic enzyme gene expression data can support the metabolomic results, and also provide deeper insight into the metabolomic findings (8).

In the present study, the GC-MS-based non-targeted metabolomic technique $(9,10)$ was used to study different metabolite patterns and metabolic pathway disturbances in PTC. Based on the metabolomic results, a follow-up study was performed to understand the expression of the metabolic enzyme genes in association with PTC. By the integration of the metabolomic and metabolic enzyme gene expression data, the present study provided the first critical insight into the metabolic network that may drive tumorigenesis in PTC.

\section{Patients and methods}

Sample collection. The Ethics Committee of the First Affiliated Hospital of Nanjing Medical University (Nanjing, China) approved the study. Informed consent was obtained from each participant. Matched PTC and normal thyroid tissues were obtained from the same diagnosed PTC patients $(n=16 ; 4$ males, 12 females; age range, 19-59 years; tumor size, $1-4.2 \mathrm{~cm}$ ) during surgery. The sample size and characteristics of the population for metabolomic analysis were similar to previous metabolomic studies on PTC and other types of cancer, and as such can provide useful information on metabolic changes of cancers using tissues (2,5-7). All the patients had undergone surgical thyroidectomy at the First Affiliated Hospital of Nanjing Medical University. Histological assessment was conducted based on established criteria of the World Health Organization (11). Pathological diagnosis of all the PTC was confirmed independently by two pathologists. Tumor samples were carefully microdissected to ensure that $>90 \%$ of the analyzed tissue contained cancer cells, and normal tissues were not connected by follicular adenomas or thyroid carcinoma. None of the patients received radiation therapy or neo-adjuvant chemotherapy prior to surgery. Fresh tumor tissue and corresponding normal thyroid were washed with phosphate-buffered saline following removal and snap-frozen in liquid nitrogen during surgery, and were subsequently stored at $-80^{\circ} \mathrm{C}$ until analysis.

Chemicals. ${ }^{13} \mathrm{C}_{6}$-Leucine was purchased from Shanghai Intechem Technology Co., Ltd. (Shanghai, China). Bis-(trimethylsilyl)trifluoroacetamide (BSTFA) with $1 \%$ trimethylchlorosilane (TMCS) and methoxylamine were obtained from Sigma-Aldrich (St. Louis, MO, USA). Water was purified by the Milli-Q Reagent Water System (Millipore, Billerica, MA, USA).

Sample preparation. For GC-MS analysis, the sample preparation was carried out according to a previous method (10).
Each 40-mg tissue was transferred to a centrifuge tube. An ice-cold mixture $(800 \mu \mathrm{l})$ of chloroform-methanol-water $(2: 5: 2, \mathrm{v} / \mathrm{v} / \mathrm{v})$ and $100 \mu \mathrm{l}$ of ${ }^{13} \mathrm{C}_{6}$-leucine, as the internal standard $(100 \mu \mathrm{g} / \mathrm{ml})$, were added to each sample and the mixture was homogenized in an ice-water bath. The samples were subsequently centrifuged at $14,000 \mathrm{x}$ g for $15 \mathrm{~min}$ at $4^{\circ} \mathrm{C}$ and $700 \mu \mathrm{l}$ of the supernatant was collected separately from each sample into a new tube. Following the addition of $500 \mu \mathrm{l}$ methanol into each original tube, the solution was vortex-mixed, and centrifuged at $14,000 \mathrm{x} \mathrm{g}$ for $15 \mathrm{~min}$ at $4^{\circ} \mathrm{C}$. The supernatant $(500 \mu \mathrm{l})$ was collected from each original tube and transferred into the corresponding new tube separately and the solution was vortex-mixed. The solution $(200 \mu \mathrm{l})$ from each new tube was transferred to a screw vial with PTFE-lined screw caps and evaporated to dryness under a stream of nitrogen gas. Subsequently, methoximation was carried out by dissolving the samples in $30 \mu \mathrm{l}$ of methoxamine solution $(20 \mathrm{mg} / \mathrm{ml}$ in pyridine) and incubating them at $37^{\circ} \mathrm{C}$ for $90 \mathrm{~min}$. Following this, $30 \mu \mathrm{BSTFA}$ with $1 \%$ TMCS was added to each vial, and the mixture was incubated for $60 \mathrm{~min}$ at a temperature of $70^{\circ} \mathrm{C}$. Following derivatization and cooling to room temperature, the derivative $(1 \mu \mathrm{l})$ was injected in the GC-MS for analysis.

$G C-M S$ analysis. The metabolomic profiling was performed according to previous studies $(9,10)$. The GC-MS instrument used for metabolite profiling was an Agilent 7890A/5975C GC-MS system with an HP-5ms fused silica capillary column (30 $\mathrm{m} \times 250 \mu \mathrm{m}, 0.25 \mu \mathrm{m}$ ). Helium (purity $>99.999 \%$ ) was used as a carrier gas with a flow rate of $1.0 \mathrm{ml} / \mathrm{min}$, and a $1 \mu 1$ sample was injected at a splitless mode. The temperature of injection was set to $280^{\circ} \mathrm{C}$. The column temperature was first kept at $80^{\circ} \mathrm{C}$ for $2 \mathrm{~min}$, increased to $320^{\circ} \mathrm{C}$ at a rate of $10^{\circ} \mathrm{C} / \mathrm{min}$ and maintained at $320^{\circ} \mathrm{C}$ for $6 \mathrm{~min}$. The detector was a quadrupole mass spectrometer and the temperatures of the ion source and quadrupole were 230 and $150^{\circ} \mathrm{C}$, respectively. The electron energy was operated at $70 \mathrm{eV}$. The data were acquired in full scan mode from m/z 50 to 600 . All the samples were analyzed randomly to avoid complications associated with the injection order. The GC-MS analysis was performed within $24 \mathrm{~h}$ to ensure the stability of instrument performance and derivatives. One script of $\mathrm{xcms}$ package was run under $\mathrm{R}$ analytical platform (R Core Team, Vienna, Austria; http://www.R-project. org/) to preprocess these raw GC-MS data, including baseline filtering, peak identification, integration, retention time correction, peak alignment and mass tag correlation. Prior to analysis, the set of data were normalized by dividing the spectral intensity of each metabolite by the sum of all the metabolites in that spectrum. This was performed to account for any differences in global sample concentrations resulting from metabolomic analysis (12). A data matrix of each sample was obtained for characterizing the biochemical pattern, and subsequently employed for correlation analysis and pattern recognition. The differential metabolites were identified by searching the NIST library and author-constructed standard library with a similarity of $>80 \%$, and were verified by available reference compounds $(9,13)$.

Reverse transcription-quantitative polymerase chain reaction (RT-qPCR) analysis. Total RNA was extracted from snap-frozen tissue fragments using TRIzol (Invitrogen, Carlsbad, CA, 
Table I. Primers for reverse transcription-quantitative polymerase chain reaction.

\begin{tabular}{|c|c|c|}
\hline Target gene & Primer sequences & Product length (bp) \\
\hline$G 6 P D$ & $\begin{array}{l}\text { Sense: 5'-ACAGAGTGAGCCCTTCTTCAA-3' } \\
\text { Antisense: 5'-GGAGGCTGCATCATCGTACT-3' }\end{array}$ & 106 \\
\hline$P G K 1$ & $\begin{array}{l}\text { Sense: 5'-TGGACGTTAAAGGGAAGCGG-3' } \\
\text { Antisense: 5'-GCTCATAAGGACTACCGACTTGG-3' }\end{array}$ & 152 \\
\hline$L D H A$ & $\begin{array}{l}\text { Sense: 5'-ATGGCAACTCTAAAGGATCAGC-3' } \\
\text { Antisense: 5'-CCAACCCCAACAACTGTAATCT-3' }\end{array}$ & 86 \\
\hline PHGDH & $\begin{array}{l}\text { Sense: 5'-CTGCGGAAAGTGCTCATCAGT-3' } \\
\text { Antisense: 5'-TGGCAGAGCGAACAATAAGGC-3' }\end{array}$ & 154 \\
\hline PTGS2 & $\begin{array}{l}\text { Sense: 5'-CTGGCGCTCAGCCATACAG-3' } \\
\text { Antisense: 5'-CGCACTTATACTGGTCAAATCCC-3' }\end{array}$ & 94 \\
\hline$\beta$-actin & $\begin{array}{l}\text { Sense: 5'-GTGGACATCCGCAAAGAC-3' } \\
\text { Antisense: 5'-GAAAGGGTGTAACGCAACT-3' }\end{array}$ & 303 \\
\hline
\end{tabular}
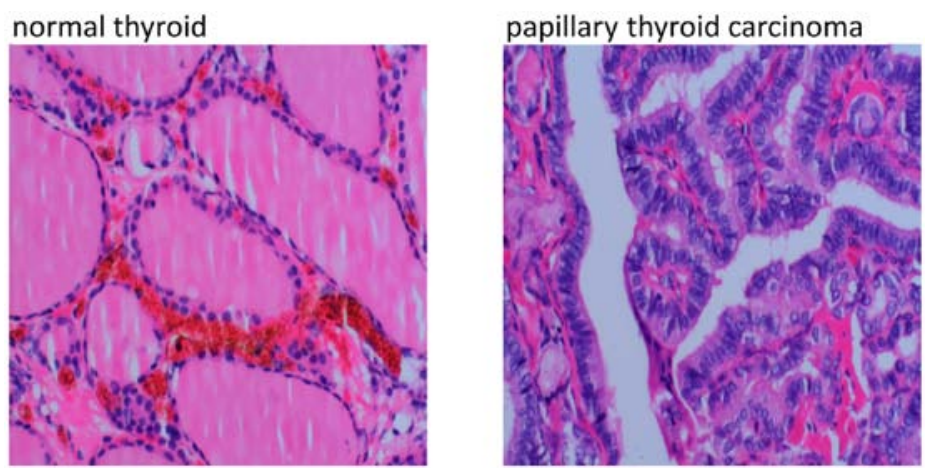

Figure 1. Hematoxylin and eosin-stained representative regions of normal thyroid and papillary thyroid carcinoma tissue from one patient (original magnification, $\mathrm{x} 200)$.

USA), according to the manufacturer's protocol. Reverse transcription was performed using the PrimeScript RT reagent kit (Takara, Dalian, China) in accordance with the manufacturer's protocol. All RT-qPCR reactions were carried out on the ABI 7900 HT Fast Real-Time system (Applied Biosystems, Foster City, CA, USA) using SYBR-Green PCR Master Mix reagent kits (Takara) according to the manufacturer's protocol for quantification of gene expression. Human-specific primers for the genes of interest are listed in Table I. All PCR was performed in triplicate, and the specificity of the PCR products was confirmed using melting curve analyses. The $2^{-\Delta \Delta C t}$ method was used to calculate the relative expression (14). The reference gene $\beta$-actin was used as an internal control. The levels of the glucose-6-phosphate dehydrogenase (G6PD), phosphoglycerate kinase $1(P G K 1)$, lactate dehydrogenase A $(L D H A)$, phosphoglycerate dehydrogenase $(P H G D H)$ and prostaglandin-endoperoxide synthase 2 (PTGS2) genes were normalized relative to the expression levels of the gene $\beta$-actin.

Statistical analysis. Multivariate statistical analysis was performed with SIMCA-P (Umetrics, Umeå, Sweden) with mean centering and unit variance scaling applied (15). To guard against over-fitting, the default 7-round cross-validation was applied with $1 / 7$ th of the samples being excluded from the mathematical model in each round. An orthogonal partial least-squares discriminate analysis (OPLS-DA) model was taken as a coefficient for peak selection (16). The significant metabolites were identified using OPLS-DA to identify metabolites with a variable importance in the projection (VIP) of $>1$ and by t-test $(\mathrm{P}<0.05)(17)$. Paired t-tests were used to compare the mRNA levels of metabolic enzyme genes.

\section{Results}

Metabolomic profiling. Fig. 1 shows the magnified representative regions of normal thyroid and PTC from one patient. Representative GC-MS total ion chromatograms of samples from the control and case groups are shown in Fig. 2. The data showed a stable retention time with no drift in all the peaks. To further validate the quality of analysis, the variation of ${ }^{13} \mathrm{C}_{6}$-leucine spectral intensities was analyzed in all the samples, and identified that the coefficient of variation \% was $11.3 \%$. All these data reflect the stability of GC-MS analysis and the reliability of the metabolomic data $(9,10)$.

Principal component analysis (PCA). PCA is an unsupervised, reductive statistical modeling technique that separates samples based on their differences from each other. PCA was used to 

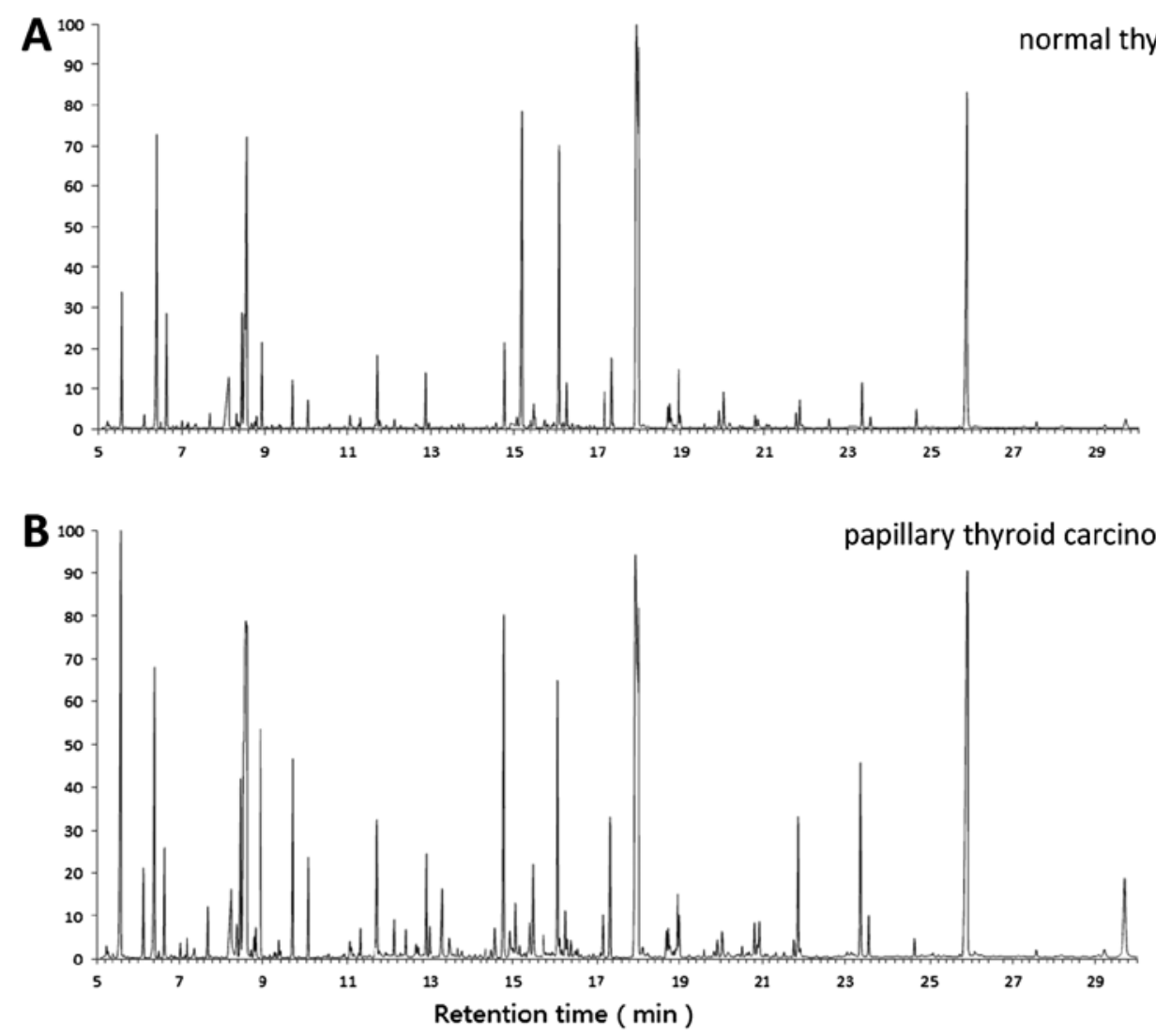

Figure 2. Representative gas chromatography coupled with mass spectrometry total ion chromatograms of the samples following chemical derivatization. (A) Normal thyroid. (B) Papillary thyroid carcinoma.

A

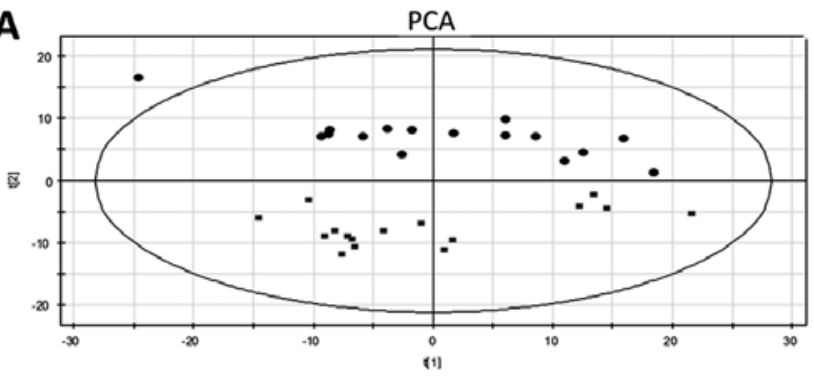

B

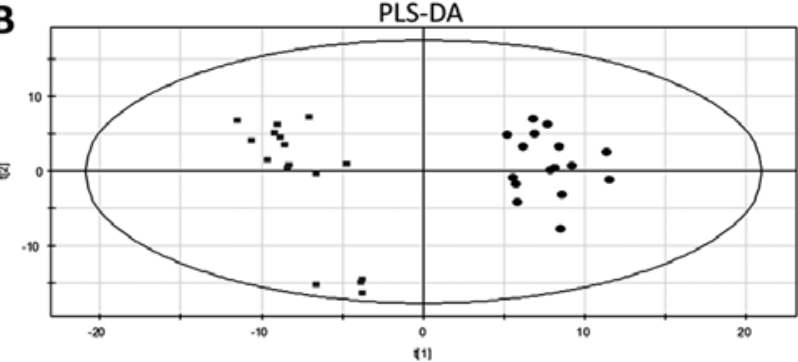

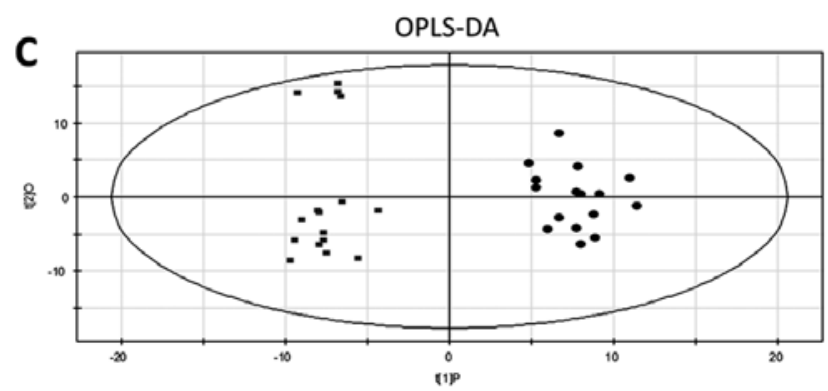

Figure 3. Comparison of papillary thyroid carcinoma (PTC) and normal thyroid with multivariate statistical analysis models. PTC (cycle), normal thyroid (square). (A) Principal component analysis (PCA) score plots discriminating PTC specimens from normal specimens based on gas chromatography coupled with mass spectrometry (GC-MS) metabolites. (B) Partial least-squares discriminant analysis (PLS-DA) scores plot discriminating PTC specimens from normal specimens based on GC-MS metabolites. (C) Orthogonal partial least-squares discriminate analysis (OPLS-DA) scores plot discriminating PTC specimens from normal specimens based on GC/MS metabolites.

identify overall metabolic differences in the data set (Fig. 3A). The normal thyroid and tumor samples were clearly separated, indicating that the metabolome was significantly changed in
PTC tissue and that metabolomic analysis acquired enough metabolome information to explain the metabolic disturbance in PTC. 
Partial least-squares discriminate analysis. Partial least-squares discriminant analysis (PLS-DA) is similar to PCA but is supervised, allowing for the definition of classes prior to performing the test. This allowed the differences between the two classes to become apparent (Fig. 3B). $R^{2} Y$ of PLS-DA was 0.98 , indicating that the model presents a high degree of goodness of fit, and the $Q^{2}$ of PLS-DA was 0.88 , indicating that the model presents a high degree of cross validation predictive ability.

OPLS-DA. OPLS-DA maximizes the variation between the specified groups and minimizes the variation between the individual replicates. Therefore, it highlights the important differences between the two compared sample classes. VIP provides an indication of each metabolite's significance to that model. A metabolite with a VIP of $>1$ is considered to have a statistically significant contribution to the model (17). Similar to the PLS-DA model, the OPLS-DA model provided optimal modeling and predictive abilities $\left(R^{2} Y=0.96, Q^{2}=0.89\right)$, achieving a distinct separation between the metabolite profiles of the two groups (Fig. 3C).

Differential metabolites associated with PTC. Differential metabolites associated with tumorigenesis derived from the OPLS-DA mode of GC-MS analysis with t-test are shown in Table II. The metabolites in carbohydrate metabolism, including glucose, fructose, galactose, mannose, 2-keto-D-gluconic acid and rhamnose, consistently decreased, while metabolites in nucleotide metabolism, including malonic acid and inosine, and lipid metabolism, including cholesterol and arachidonic acid, significantly altered correspondingly. The classification of the chemicals and mapping of the metabolites into general biochemical pathways, as illustrated in the Kyoto Encyclopedia of Genes and Genomes (http://www.genome.jp/kegg/), suggested the possible increased carbohydrate-derived flux into the glycolysis and pentose phosphate pathway and increased oncogenic lipid synthesis in PTC (Fig. 4).
Table II. Differential metabolites associated with PTC derived from the OPLS-DA mode of gas chromatography coupled with mass spectrometry analysis with t-test.

\begin{tabular}{lccc}
\hline Metabolites & VIP-value $^{\mathrm{a}}$ & P-value & Fold-change $^{\mathrm{c}}$ \\
\hline Arachidonic acid & 1.72 & $1.14 \times 10^{-3}$ & 0.50 \\
2-keto-D-gluconic acid & 2.25 & $2.87 \times 10^{-6}$ & 0.26 \\
Malonic acid & 1.36 & $1.96 \times 10^{-2}$ & 0.16 \\
Glucose & 2.06 & $3.82 \times 10^{-5}$ & 0.31 \\
Rhamnose & 1.79 & $5.70 \times 10^{-4}$ & 0.48 \\
Fructose & 1.52 & $4.83 \times 10^{-3}$ & 0.27 \\
Mannose & 1.63 & $2.19 \times 10^{-3}$ & 0.22 \\
Galactose & 1.55 & $3.62 \times 10^{-3}$ & 0.19 \\
Phenylalanine & 1.42 & $1.85 \times 10^{-2}$ & 2.02 \\
N6-Acetyl-L-lysine & 1.13 & $4.06 \times 10^{-2}$ & 1.58 \\
Inosine & 2.21 & $5.39 \times 10^{-6}$ & 3.53 \\
Hydroxyproline & 1.53 & $5.80 \times 10^{-3}$ & 9.88 \\
Benzoic acid & 1.78 & $6.19 \times 10^{-4}$ & 1.18 \\
Cholesterol & 1.12 & $4.54 \times 10^{-2}$ & 1.32 \\
Pelargonic acid & 1.62 & $2.25 \times 10^{-3}$ & 1.33
\end{tabular}

avariable importance in the projection (VIP) was obtained from OPLS-DA with a threshold of $1.0 .{ }^{\text {b }} \mathrm{P}$-value was calculated with t-test. ${ }^{\mathrm{c}}$ Fold change with a value $>1$ indicates a relatively higher concentration in the PTC tissue when compared with normal tissue, whereas a value $<1$ means a relatively lower concentration in PTC tissue. PTC, papillary thyroid carcinoma; OPLS-DA, orthogonal partial least-squares discriminate analysis.

Metabolic enzyme gene expression change and metabolic network in PTC. A combination of metabolomic and associated mRNA data has been proven to be useful in providing deeper insight into the metabolic changes (18).

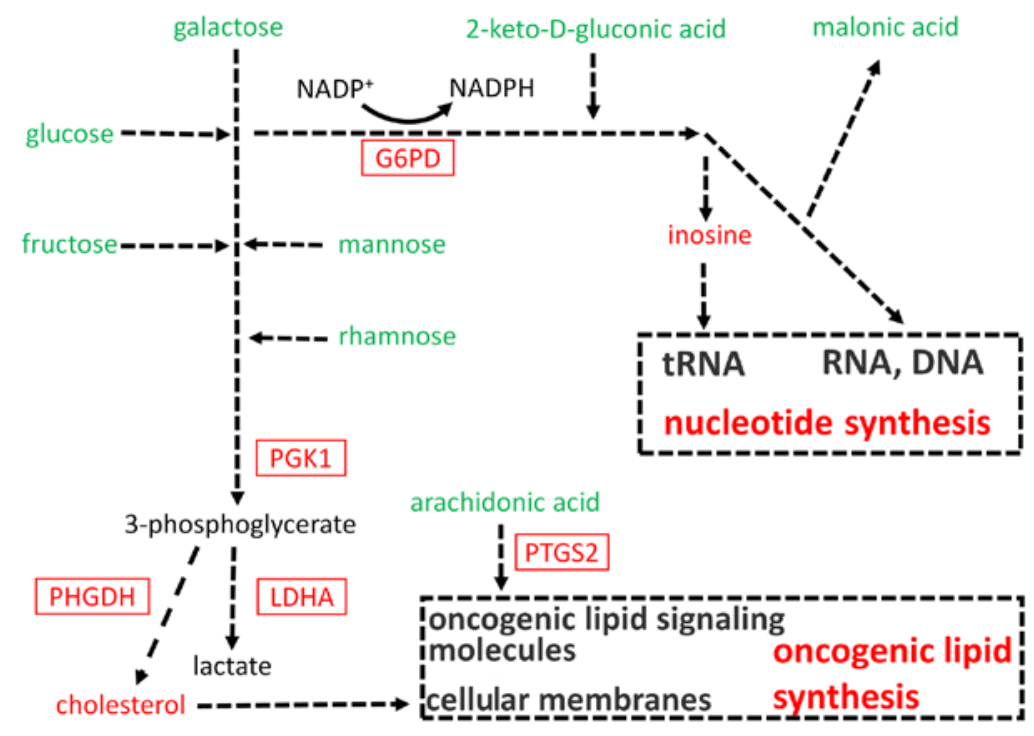

Figure 4. Dysregulated metabolic network in papillary thyroid carcinoma (PTC). Green indicates a decrease in PTC; red indicates an increase in PTC; black indicates no available data. G6PD, glucose-6-phosphate dehydrogenase; PGK1, phosphoglycerate kinase 1; LDHA, lactate dehydrogenase A; PHGDH, phosphoglycerate dehydrogenase; PTGS2, prostaglandin-endoperoxide synthase 2. 

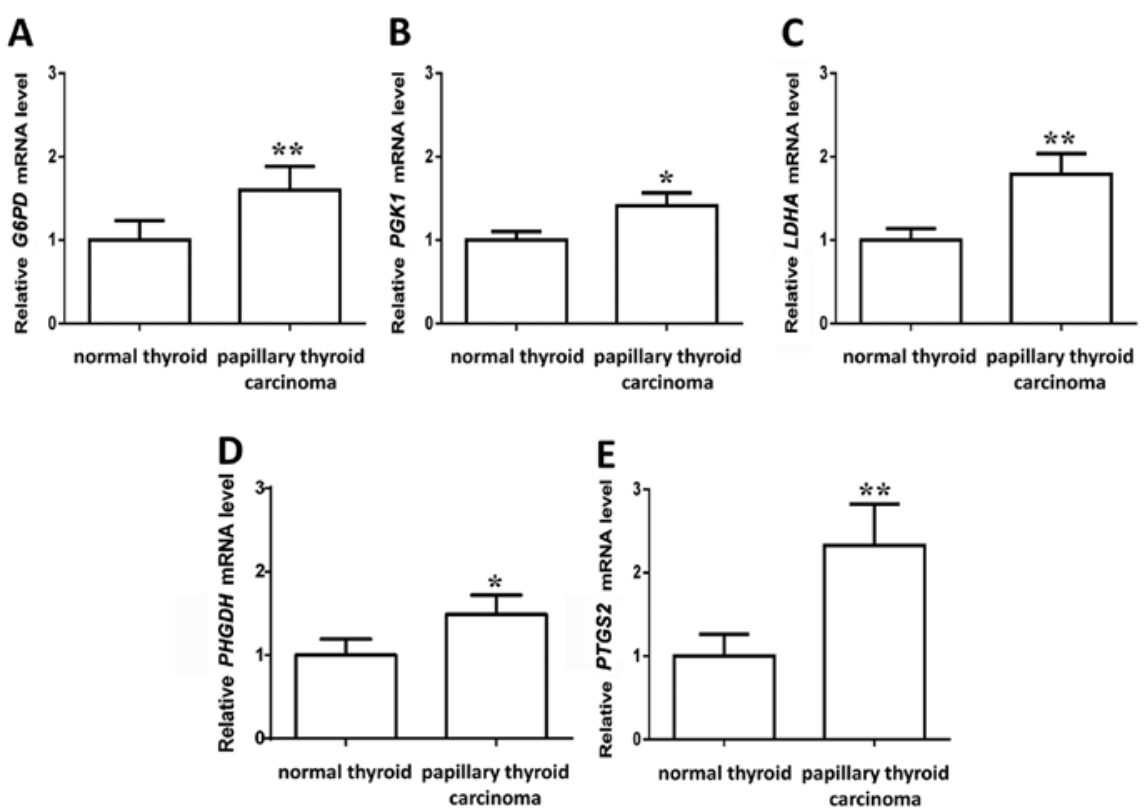

Figure 5. Comparison of the mRNA levels of the metabolic enzyme genes in papillary thyroid carcinoma and normal thyroid. Each bar represents the mean \pm standard error of the mean, $\mathrm{n}=16$ for each group. The data shown are representative of the results of three independent reverse transcription-quantitative polymerase chain reaction analysis. Each value was normalized to the mRNA expression of $\beta$-actin, and a summary of the results is expressed as percentage of control mRNA levels. ${ }^{*} \mathrm{P}<0.05,{ }^{* *} \mathrm{P}<0.01$. (A) Glucose-6-phosphate dehydrogenase $(G 6 P D)$; (B) phosphoglycerate kinase 1 (PGK1); (C) lactate dehydrogenase A $(L D H A)$; (D) phosphoglycerate dehydrogenase $(P H G D H)$; and (E) prostaglandin-endoperoxide synthase $2(P T G S 2)$.

In order to validate the metabolite changes and further delineate the metabolic network in PTC, the expression of several critical metabolic enzyme genes was determined in the proposed metabolic pathway by analyzing the mRNA level. The mRNA levels of gene G6PD encoding glucose-6-phosphate dehydrogenase, which is the committed step of the pentose phosphate pathway, significantly increased in PTC (Figs. 4 and $5 \mathrm{~A})$. In the glycolysis pathway, the mRNA levels of the gene $P G K 1$ encoding phosphoglycerate kinase, which is a crucial enzyme in the glycolysis cycle and catalyzes the formation of 3-phosphoglycerate, significantly increased in PTC (Figs. 4 and $5 \mathrm{~B}$ ). The mRNA levels of the gene $L D H A$ encoding lactate dehydrogenase, significantly increased in PTC (Figs. 4 and 5C), supporting the finding of increased glycolysis in PTC. As mRNA levels of the PGK1 significantly increased (Figs. 4 and 5B), the metabolism of 3-phosphoglycerate was focused on, which can provide acetyl-CoA for cholesterol synthesis. The mRNA levels of the gene PHGDH encoding phosphoglycerate dehydrogenase increased in PTC (Fig. 5D), which can finally produce acetyl-CoA to support lipogenesis, indicating that the 3-phosphoglycerate metabolic pathway may generate precursors for the downstream production of cholesterol. The relative levels of arachidonic acid decreased in PTC, which is the precursor of oncogenic lipid. The mRNA levels of gene PTGS2 encoding prostaglandin-endoperoxide synthase 2 increased (Fig. 5E) in PTC, indicating an increased consumption of arachidonic acid to form the oncogenic lipid in PTC. The metabolic network that may drive tumorigenesis in PTC is summarized in Fig. 4.

\section{Discussion}

Although the metabolic changes have been studied in different cancers (19-21), the metabolic changes that drive tumorigenesis in PTC remain to be elucidated. Notable consistent decreases in a wide range of metabolites, including glucose, fructose, galactose, mannose, 2-keto-D-gluconic acid and rhamnose in the upper section of the glycolysis and pentose phosphate pathway, were observed in PTC samples. The increased $L D H A$ expression is in accordance with previous studies using the NMR-based metabolomic technique, which has consistently revealed that the lactate levels increased in PTC $(6,7)$. These results, including metabolomic and gene expression data, consistently indicate upregulation of the glycolysis and pentose phosphate pathway by high consumption of glucose, fructose, galactose, mannose, 2-keto-D-gluconic acid and rhamnose in PTC.

The upregulation of the pentose phosphate pathway by the increased G6PD expression and higher levels of consumption of glucose, galactose and 2-keto-D-gluconic acid in tumor cells provides important precursors (pentoses, 5-carbon sugars) of nucleotide synthesis, such as DNA and RNA. Accordingly, the production of the downstream metabolite, inosine, significantly increased in PTC (Table II and Fig. 4). Inosine is commonly found in tRNAs and is essential for correct translation of the genetic code. The decrease of the associated levels of malonic acid in PTC indicates possible diversion into the uridine monophosphate synthesis pathway in PTC to increase nucleotide synthesis (22). Therefore, these data suggest a link between the upregulation of the pentose phosphate pathway and increased nucleotide synthesis in PTC to initiate and maintain tumorigenesis.

Aside from pentoses, the pentose phosphate pathway is a process that also generates nicotinamide adenine dinucleotide phosphate (NADPH). Ribonucleotide reductase (RNR) is an enzyme that catalyzes the formation of deoxyribonucleotides from ribonucleotides (23). Deoxyribonucleotides are used in the synthesis of DNA, and RNR has a critical role in regulating 
the total rate of DNA synthesis. The ultimate reductant of this reduction system is NADPH. Therefore, the upregulation of pentose phosphate pathway not only provides more precursors for DNA synthesis, but also aids in the generation of DNA by RNR in PTC. By contrast, a recent study has shown that control of the intracellular reactive oxygen species concentrations is critical for lung cancer cell survival (24). NADPH can also be used as the primary reducing power, which may aid in proliferation and lung cancer cell growth (24).

In the present study, the 3-phosphoglycerate metabolic pathway may generate precursors for the increased cholesterol production in PTC. Cholesterol is an essential structural component of human cell membranes, and it is reported that cholesterol increases in colorectal cancer tissue (25). In PTC tissue, the increase of cholesterol may drive rapid cell growth and proliferation. It is also noteworthy that the conversion of 3 -phosphoglycerate generates glycine. Glycine is a precursor of numerous important molecules that are required for cell growth, such as purines, protein, glutathione and 1-carbon units as 5,10-methylenetetrahydrofolate (26).

In PTC, significantly decreased levels of arachidonic acid were observed. Arachidonic acid is the precursor of prostaglandins, which are a class of oncogenic lipid signaling molecules (27). PTGS2 catalyzes the conversion of arachidonic acid to prostaglandins, and it is reported that PTGS2 can be induced at sites of inflammation by cytokines, growth factors, tumor promoters and other agents in colorectal cancer (28). In the present study, the mRNA levels of PTGS 2 encoding prostaglandin-endoperoxide synthase 2 increased in PTC (Figs. 4 and 5E), and an increased consumption of arachidonic acid was observed, which forms the oncogenic lipid in PTC. Therefore, the decrease of arachidonic acid can be explained by an increased generation of prostaglandins in PTC.

Although several NMR-based metabolomic studies on thyroid carcinomas have been reported (5-7), the GC-MS-based metabolomic analysis of thyroid carcinomas has never been reported. In the present GC-MS-based metabolomic study, the metabolomic data could establish models with high degrees of goodness-of-fit and cross validation predictive ability, and the normal thyroid and tumor samples could be clearly separated, suggesting this GC-MS-based metabolomic approach has a significant potential in improving the diagnostic efficacy of PTC in conjunction with current diagnostics. Further metabolomic studies focusing on the diagnosis of thyroid cancers should be conducted using urine and blood samples and require the involvement of benign masses, as well as other pathological types of thyroid cancers to provide non-invasive and rapid diagnostic techniques for thyroid cancers (29).

In addition to the dysregulated metabolism pathway in PTC, there was a change of N6-Acetyl-L-lysine. N6-Acetyl-L-lysine is an acetylated amino acid that has critical roles in regulating gene transcription, cell-cycle progression, apoptosis, DNA repair and cytoskeletal organization. The observed increase of N6-Acetyl-L-lysine indicates that an intracellular signaling mechanism by post-translational lysine-acetylation may be involved in the pathogenesis of PTC.

In conclusion, through using a novel integrated analysis of metabolome and metabolic enzyme gene expression in PTC, to the best of our knowledge the metabolite patterns and metabolic networks in PTC have been shown for the first time.
Various metabolites that may be used for increased synthesis of nucleotide and oncogenic lipid were identified in PTC via the increased expression of associated metabolic enzyme genes, which may contribute to the pathogenesis of PTC. The present study provides an understanding of dysregulated metabolism in PTC and identifies potential avenues for the therapeutic intervention for this disease.

\section{Acknowledgements}

The present study was supported by the National Natural Science Foundation of China (no. 81370920); the Natural Science Foundation of Jiangsu Province (no. BK20131110); the Project of 'Six Talents Peak of Jiangsu province (no. 2013WSN-023)'; the Open Project Funding of Jiangsu Province Key Research for Molecular and Functional Laboratory (no. PYZX 2012004); the Jiangyin City Science and Technology Founded Project (2014); and the Jiangsu Province Official Hospital Scientific Research Initial Funding (RPF201501).

\section{References}

1. Wang Z, Klipfell E, Bennett BJ, Koeth R, Levison BS, Dugar B Feldstein AE, Britt EB, Fu X, Chung YM, et al: Gut flora metabolism of phosphatidylcholine promotes cardiovascular disease. Nature 472: 57-63, 2011.

2. Sreekumar A, Poisson LM, Rajendiran TM, Khan AP, Cao Q Yu J, Laxman B, Mehra R, Lonigro RJ, Li Y, et al: Metabolomic profiles delineate potential role for sarcosine in prostate cancer progression. Nature 457: 910-914, 2009.

3. Tritten L, Keiser J, Godejohann M, Utzinger J, Vargas M, Beckonert O, Holmes E and Saric J: Metabolic profiling framework for discovery of candidate diagnostic markers of malaria. Sci Rep 3: 2769, 2013.

4. Wang L, Chen J, Chen L, Deng P, Bu Q, Xiang P, Li M, Lu W, $\mathrm{Xu} \mathrm{Y,} \mathrm{Lin} \mathrm{H,} \mathrm{et} \mathrm{al:} 1 \mathrm{H}-\mathrm{NMR}$ based metabonomic profiling of human esophageal cancer tissue. Mol Cancer 12: 25, 2013.

5. Jordan KW, Adkins CB, Cheng LL and Faquin WC: Application of magnetic-resonance-spectroscopy- based metabolomics to the fine-needle aspiration diagnosis of papillary thyroid carcinoma. Acta Cytol 55: 584-589, 2011.

6. Miccoli P, Torregrossa L, Shintu L, Magalhaes A, Chandran J, Tintaru A, Ugolini C, Minuto MN, Miccoli M, Basolo F, et al: Metabolomics approach to thyroid nodules: A high-resolution magic-angle spinning nuclear magnetic resonance-based study. Surgery 152: 1118-1124, 2012.

7. Deja S, Dawiskiba T, Balcerzak W, Orczyk-Pawiłowicz M, Głód M, Pawełka D and Młynarz P: Follicular adenomas exhibit a unique metabolic profile. ${ }^{1} \mathrm{H}$ NMR studies of thyroid lesions. PLoS One 8: e84637, 2013.

8. Chen M, Zhou K, Chen X, Qiao S, Hu Y, Xu B, Xu B, Han X, Tang R, Mao Z, et al: Metabolomic analysis reveals metabolic changes caused by bisphenol A in rats. Toxicol Sci 138: 256-267, 2014.

9. Wu H, Xue R, Lu C, Deng C, Liu T, Zeng H, Wang Q and Shen X: Metabolomic study for diagnostic model of oesophageal cancer using gas chromatography/mass spectrometry. J Chromatogr B Analyt Technol Biomed Life Sci 877: 3111-3117, 2009.

10. Wu H, Xue R, Tang Z, Deng C, Liu T, Zeng H, Sun Y and Shen X: Metabolomic investigation of gastric cancer tissue using gas chromatography/mass spectrometry. Anal Bioanal Chem 396: 1385-1395, 2010.

11. DeLellis RA, Lloyd RV, Heitz PU and Eng C (eds): Pathology and Genetics of Tumours of Endocrine Organs. Vol 8. 3rd edition. World Health Organization, Lyon, France, IARC Press, 2004.

12. Booth SC, Workentine ML, Wen J, Shaykhutdinov R, Vogel HJ, Ceri H, Turner RJ and Weljie AM: Differences in metabolism between the biofilm and planktonic response to metal stress. J Proteome Res 10: 3190-3199, 2011.

13. Qiu Y, Cai G, Zhou B, Li D, Zhao A, Xie G, Li H, Cai S, Xie D, Huang C, et al: A distinct metabolic signature of human colorectal cancer with prognostic potential. Clin Cancer Res 20: 2136-2146, 2014 
14. Ferreira ID, Rosário VE and Cravo PV: Real-time quantitative PCR with SYBR Green I detection for estimating copy numbers of nine drug resistance candidate genes in Plasmodium falciparum. Malar J 5: 1, 2006.

15. Slupsky CM, Steed H, Wells TH, Dabbs K, Schepansky A, Capstick V, Faught W and Sawyer MB: Urine metabolite analysis offers potential early diagnosis of ovarian and breast cancers. Clin Cancer Res 16: 5835-5841, 2010.

16. Chen T, Xie G, Wang X, Fan J, Qiu Y, Zheng X, Qi X, Cao Y, Su M, Wang X, et al: Serum and urine metabolite profiling reveals potential biomarkers of human hepatocellular carcinoma. Mol Cell Proteomics 10: M110 004945, 2011.

17. Cai Z, Zhao JS, Li JJ, Peng DN, Wang XY, Chen TL, Qiu YP, Chen PP, Li WJ, Xu LY, et al: A combined proteomics and metabolomics profiling of gastric cardia cancer reveals characteristic dysregulations in glucose metabolism. Mol Cell Proteomics 9: 2617-2628, 2010.

18. Parman T, Bunin DI, Ng HH, McDunn JE, Wulff JE, Wang A, Swezey R, Rasay L, Fairchild DG, Kapetanovic IM, et al: Toxicogenomics and metabolomics of pentamethylchromanol (PMCol)-induced hepatotoxicity. Toxicol Sci 124: 487-501, 2011.

19. Goedert JJ, Sampson JN, Moore SC, Xiao Q, Xiong X, Hayes RB, Ahn J, Shi J and Sinha R: Fecal metabolomics: Assay performance and association with colorectal cancer. Carcinogenesis 35: 2089-2096, 2014.

20. Mathé EA, Patterson AD, Haznadar M, Manna SK, Krausz KW, Bowman ED, Shields PG, Idle JR, Smith PB, Anami K, et al: Noninvasive urinary metabolomic profiling identifies diagnostic and prognostic markers in lung cancer. Cancer Res 74 3259-3270, 2014.

21. Zhang AH, Sun H, Qiu S and Wang XJ: Metabolomics in noninvasive breast cancer. Clin Chim Acta 424: 3-7, 2013.

22. Hayaishi $\mathrm{O}$ and Kornberg A: Metabolism of cytosine, thymine, uracil, and barbituric acid by bacterial enzymes. J Biol Chem 197: 717-732, 1952.
23. Elledge SJ, Zhou Z and Allen JB: Ribonucleotide reductase: Regulation, regulation, regulation. Trends Biochem Sci 17: 119-123, 1992.

24. Anastasiou D, Poulogiannis G, Asara JM, Boxer MB, Jiang JK, Shen M, Bellinger G, Sasaki AT, Locasale JW, Auld DS, et al: Inhibition of pyruvate kinase M2 by reactive oxygen species contributes to cellular antioxidant responses. Science 334: 1278-1283, 2011

25. Chan EC, Koh PK, Mal M, Cheah PY, Eu KW, Backshall A, Cavill R, Nicholson JK and Keun HC: Metabolic profiling of human colorectal cancer using high-resolution magic angle spinning nuclear magnetic resonance (HR-MAS NMR) spectroscopy and gas chromatography mass spectrometry (GC/MS). J Proteome Res 8: 352-361, 2009.

26. Lamers Y, Williamson J, Ralat M, Quinlivan EP, Gilbert LR, Keeling C, Stevens RD, Newgard CB, Ueland PM, Meyer K, et al: Moderate dietary vitamin B-6 restriction raises plasma glycine and cystathionine concentrations while minimally affecting the rates of glycine turnover and glycine cleavage in healthy men and women. J Nutr 139: 452-460, 2009.

27. Nomura DK, Long JZ, Niessen S, Hoover HS, Ng SW and Cravatt BF: Monoacylglycerol lipase regulates a fatty acid network that promotes cancer pathogenesis. Cell 140: 49-61, 2010.

28. Wang D, Xia D and Dubois RN: The crosstalk of PTGS2 and EGF signaling pathways in colorectal cancer. Cancers (Basel) 3: 3894-3908, 2011.

29. Brindle JT, Antti H, Holmes E, Tranter G, Nicholson JK, Bethell HW, Clarke S, Schofield PM, McKilligin E, Mosedale DE, et al: Rapid and noninvasive diagnosis of the presence and severity of coronary heart disease using 1H-NMR-based metabonomics. Nat Med 8: 1439-1444, 2002. 